\title{
Dynamical Behavior of Hybrid Propagation of Computer Viruses
}

\author{
Qingyi Zhu $\mathbb{D}^{1,2}$ Pingfan Xiang, ${ }^{2}$ Xuhang Luo $\mathbb{D}^{1},{ }^{1}$ and Chenquan Gan $\mathbb{D}^{3}$ \\ ${ }^{1}$ School of Cyber Security and Information Law, Chongqing University of Posts and Telecommunications, \\ Chongqing 400065, China \\ ${ }^{2}$ School of Computer Science and Technology, Chongqing University of Posts and Telecommunications, Chongqing 400065, China \\ ${ }^{3}$ School of Communication and Information Engineering, Chongqing University of Posts and Telecommunications, \\ Chongqing 400065, China
}

Correspondence should be addressed to Chenquan Gan; gcq2010cqu@163.com

Received 3 November 2021; Revised 1 January 2022; Accepted 12 January 2022; Published 9 February 2022

Academic Editor: Mamoun Alazab

Copyright () 2022 Qingyi Zhu et al. This is an open access article distributed under the Creative Commons Attribution License, which permits unrestricted use, distribution, and reproduction in any medium, provided the original work is properly cited.

Considering the horizontal and vertical propagation of computer viruses over the Internet, this article proposes a hybrid susceptible-latent-breaking-recovered-susceptible (SLBRS) model. Through mathematical analysis of the model, two equilibria (virus-free and virose equilibria) and their global stabilities are both proved depending on the basic reproduction number $R_{0}$, which is affected by the vertical propagation of infected computers. Moreover, the feasibility of the obtained results is verified by numerical simulations. Finally, the dependence of $R_{0}$ on system parameters and the parameters affecting the stability level of infected computers are both analyzed.

\section{Introduction}

Computer virus is a malevolent network code designed to disseminate from one device to another [1]. Even minor computer virus can wreak havoc on a system performance, consume computer memory, and cause frequent computer crashes. With all the technological advances of the 21th century, computer virus grew at a breakneck rate. Mail viruses and macroviruses that rely on the Internet to spread have emerged in large numbers. Due to the characteristics of fast-spreading, strong concealment, and great destructiveness of computer viruses, the work of anticomputer virus becomes very difficult [2], which has brought immeasurable losses to people. At the beginning of the twenty-first century, email was an important way for computer viruses to spread. The Medsa virus and the love letter virus spread rapidly around the world via email. In 2017, the WannaCry ransomware quickly infected extensive computers in a short period, causing incalculable damage [3]. On April 12, 2021, KrebsonSecurity, an international information network security media, reported that hackers were selling the personal details of tens of millions of users of ParkMobile, a North American mobile parking application, on a Russian- language cybercrime forum along with screenshots of the data [4]. Later, Code Red and Nimda virus appeared one after another. These two viruses used a combination of mail transmission and active attack on server vulnerabilities, creating a new way of virus transmission and greatly increasing the speed of virus transmission in the network. Owing to the enormous harm brought by the malevolent virus attack, it is urgent to study the spreading behavior of viruses among network nodes and propose effective prevention and control strategies.

Seeing the resemblance between computer viruses and biological viruses, many researchers use epidemiological models to study the spread of network viruses. In the twentieth century, the author introduced the susceptibilityinfection-susceptibility (SIS) model in the field of network viruses [5]. Thereafter, the susceptible-exposed-infected (SEI) model was proposed by introducing latency bins. Xie [6] proposed the SEI model by considering the effect of heterogeneity of email networks on virus propagation. On this basis, increasingly popular models are being used and studied in the field of virus transmission, for example, susceptible-infected-recovered (SIR) models [7-9], susceptible-infected-recovered-susceptible (SIRS) models [10-13], 
susceptible-exposed-infected-recovered (SEIR) models [14-17], susceptible-exposed-infected-recovered-susceptible (SEIRS) models [18-21], susceptible-exposed-infectedquarantined-vaccinated (SEIQV) models [22, 23], and susceptible-exposed-infected-quarantined-recovered-susceptible (SEIQRS) models [24-26]. To explicitly compare the individual characteristics of computer virus propagation models, we have made tabular discussions in Table 1.

Based on the direction of computer virus transmission, network viruses can be divided into horizontal transmission and vertical transmission. Horizontal spreading refers to the copying and spreading of malicious programs through the spreading medium between nodes. Specifically, after a network virus infects a computer, it infects other computers associated with it through emails, web browsing, disk media, and mobile media. After these infected computers become new sources of infection, they can infect other computers associated with them in the same way as described above. Vertical transmission refers to the virus attacking the main server, making it a source of infection, and then passing it from the main server to any node. For example, Code Red and Nimda viruses actively attack server vulnerabilities through vertical transmission.

Generally speaking, in real life, once a computer is infected, it is immediately infectious. However, preceding scholars did not notice this difference between computer viruses and biological viruses, and they believed that latent computers are not infectious. The author [33] first noticed this deficiency of the previous computer virus model and proposed the susceptible-latent-breaking-susceptible (SLBS) model that was more consistent with the actual situation in 2012, and then proposed a series of improved models. Subsequently, a four-compartment model called the primitive SLBRS model was proposed by Yang [34]. By studying the safety tendency of a virus system based on security entropy, Tang proposed a new application scenario SLBRS computer virus model [32]. However, these models always focus on the horizontal propagation behavior of computer viruses.

In a computer network, some computer viruses may be transmitted vertically from the master server to any node, such as worms [35]. On the Internet, we have to pay attention to the influence of vertical transmission of computer virus on uninfected new nodes, but the literature on the vertical analysis of computer virus transmission behavior is not extensive. Considering some computer viruses may be vertical transmission from the main server to any node, Kumar [35] proposed the worms in the computer network SEIRS model of vertical transmission. However, this model ignores the fact that computer viruses might be infectious during both the latent and outbreak phases.

Given the vertical spread of computer viruses and the fact that latent computers are still infectious, a new SLBRS model is introduced to discuss the spread of network viruses in both horizontal and vertical directions from a macroperspective. Admittedly, this model is more sophisticated and reasonable than previous models, with the rapid growth of master servers. We deeply study the dynamics of this model. Qualitative analysis of the model has obtained the virus-free equilibrium and the virose equilibrium. Global stability of the SLBRS model is verified by using the global geometric method. In addition, numerical experiments illustrate the feasibility of the theoretical results, and the parameters of the system are discussed and analyzed.

The rest of this article is organized as follows: In Section 2 , we describe the SLBRS model with vertical propagation. In Section 3, we confirm a globally asymptotically stable virusfree equilibrium, and the virose equilibrium is obtained. In Section 4, we perform detailed numerical calculations to verify the local and global stability of the virose equilibrium. In Section 5, the influence of system parameters on $R_{0}$ is discussed. In Section 6, we present the numerical simulation results. Finally, Section 7 summarizes this article.

\section{Mathematical Model Expression}

In the SLBRS model with vertical propagation, the networked computers are defined as four types: susceptible nodes $(S)$, latent nodes $(L)$, breaking-out nodes $(B)$, and recovering nodes $(R)$.

Let $S, L, B$, and $R$ represent the proportion of the number of nodes in each of the above four categories among all nodes at a certain time, so we have

$$
S+L+B+R=1 .
$$

Figure 1 shows the transformation process of nodes in various states, and parameters used in the model are given in Table 2. The basic terms and assumptions given are used for the rest of this article.

\subsection{Model Assumptions.}

(1) Once newly added nodes are infected, they become contagious

(2) Within the network, nodes are connected to each other

(3) At some point, the bilinear infection rate of $S$ nodes becoming $L$ nodes is $\beta S(L+B)$

(4) Since the probabilities that the descendants of $L$ and $B$ nodes are $L$ nodes are $p$ and $q$, respectively, satisfying $0 \leq p \leq 1,0 \leq q \leq 1$, the probability that the newly accessed computers are $S$ nodes is $\xi-p \xi L-q \xi B$

2.2. Model Expression. Based on the previous description and assumptions, the state transition differential system of the model is 
TABLE 1: Individual characteristics and stability of computer virus propagation models.

\begin{tabular}{|c|c|c|c|c|}
\hline \multirow{2}{*}{ Contributors } & \multirow{2}{*}{ Model } & \multirow{2}{*}{ Characteristics } & \multicolumn{2}{|r|}{ Stability } \\
\hline & & & Virus-free equilibrium stability & Virose equilibrium stability \\
\hline Essouifi et al. [27] & SIR-SIS & $\begin{array}{l}\text { Two-degree networks } \\
\text { and stochastic method }\end{array}$ & $\mathrm{n} / \mathrm{a}$ & Locally asymptotically stable \\
\hline Upadhyay et al. [28] & SVEIR & Nonlinear incident rate & $\begin{array}{l}\text { Locally and globally } \\
\text { asymptotically stable }\end{array}$ & Locally and globally asymptotically stable \\
\hline Yang et al. [29] & SEIR-KS & Hopf bifurcation & $\mathrm{n} / \mathrm{a}$ & Locally asymptotically stable \\
\hline Aníbal et al. [30] & SEIR-KS & Periodic environment & $\mathrm{n} / \mathrm{a}$ & $\mathrm{n} / \mathrm{a}$ \\
\hline Zdemir et al. [31] & SEIR-KS & The kill signals & Locally asymptotically stable & Locally asymptotically stable \\
\hline Tang et al. [32] & SLBRS & Safety entropy & $\mathrm{n} / \mathrm{a}$ & Globally asymptotically stable \\
\hline This work & SLBRS & Vertical transmission & $\begin{array}{l}\text { Locally and globally } \\
\text { asymptotically stable }\end{array}$ & Locally and globally asymptotically stable \\
\hline
\end{tabular}

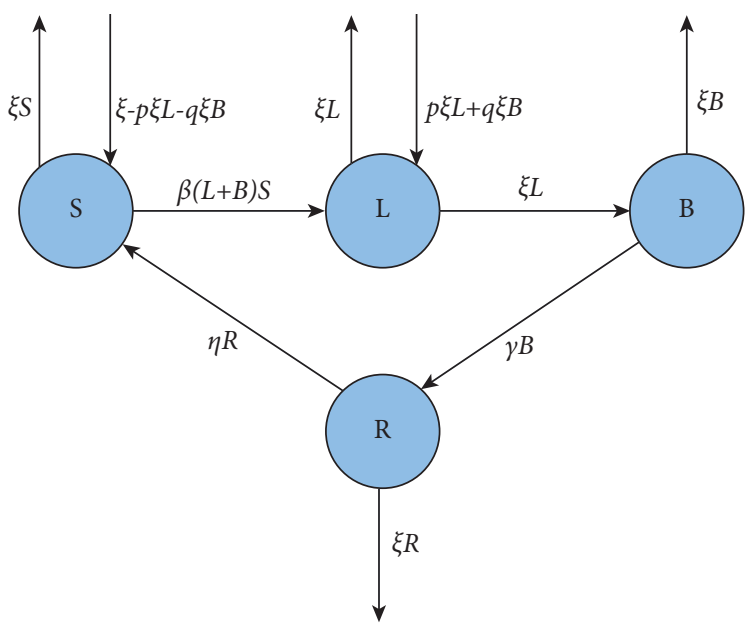

FIgURE 1: Migration blueprint of the SLBRS model.

TABLE 2: Parameters involved in the model.

\begin{tabular}{lc}
\hline Parameters & Description \\
\hline$\xi$ & The ratio of computers connected to the Internet. \\
$\beta$ & The ratio at which each $S$ computer becomes $L$ computer by contact with either $L$ computer or $B$ computer. \\
$\varepsilon$ & The rate at which $B$ computers are cured. \\
$\gamma$ & The rate at which $B$ computers are cured. \\
$\eta$ & The rate which $R$ computers lose immunity. \\
$p$ & The rate at which the descendants of the $B$ computer become the $S$ computers. \\
$q$ &
\end{tabular}

Based on the above theoretical analysis, we know that $R=1-S-L-B$, and differential equations of system (2) state can be converted into

$$
\left\{\begin{array}{l}
\frac{\mathrm{d} S}{\mathrm{~d} t}=\xi-\beta S(L+B)+\eta(1-S-L-B)-p \xi L-q \xi B-\xi S \\
\frac{\mathrm{d} L}{\mathrm{~d} t}=\beta S(L+B)-\varepsilon L+p \xi L+q \xi B-\xi L, \\
\frac{\mathrm{d} B}{\mathrm{~d} t}=\varepsilon L-\gamma B-\xi B .
\end{array}\right.
$$


The initial states of all kinds of computers in system (3), respectively, satisfy: $L(0) \geq 0, S(0) \geq 0$, and $B(0) \geq 0$. The initial value belongs to the positive invariant set:

$$
\Psi=\{(S, L, B): S+L+B \leq 1, S \geq 0, L \geq 0, B \geq 0\} .
$$

The basic regeneration number is the threshold at which a disease tends to die out or persist. We calculated the basic reproductive number is as follows:

$$
R_{0}=\frac{\beta(\xi+\gamma+\varepsilon)+(\xi+\gamma) p \xi+\varepsilon q \xi}{(\xi+\gamma)(\varepsilon+\xi)} .
$$

For one thing, from the above description, the virus-free equilibrium is calculated as $E_{0}=\left(S_{0}, L_{0}, B_{0}\right)=(1,0,0)$. In addition, data analysis of system (3) was performed to determine the unique endemic equilibrium point $E^{*}=\left(S^{*}, L^{*}, B^{*}\right)$. Specific results are as follows:

$$
\begin{aligned}
S^{*} & =\frac{(\xi+\gamma)(\epsilon+\xi)-(\xi+\gamma) p \xi-\epsilon q \xi}{\beta(\xi+\gamma+\epsilon)}, \\
L^{*} & =\frac{(\xi+\gamma)^{2}(\xi+\eta)(\epsilon+\xi)\left(R_{0}-1\right)}{(\xi+\gamma+\epsilon)[(\xi+\gamma)(\xi+\eta)+(\xi+\eta+\gamma) \epsilon]}, \\
B^{*} & =\frac{\epsilon(\xi+\gamma)(\xi+\eta)(\epsilon+\xi)\left(R_{0}-1\right)}{(\xi+\gamma+\epsilon)[(\xi+\gamma)(\xi+\eta)+(\xi+\eta+\gamma) \epsilon]} .
\end{aligned}
$$

\section{Stability of the Virus-Free Equilibrium}

Global stability of steady state revealed the basic rule of the spread of the virus. Subsequently, the global stability of the virus-free equilibrium will be derived.

Theorem 1. The virus-free equilibrium $E_{0}$ is local asymptotically stable if $R_{0} \leq 1$, but unstable if $R_{0}>1$.

Proof. We obtained the Jacobian matrix of system (3) at disease-free equilibrium as follows:

$$
J_{E_{0}}=\left[\begin{array}{ccc}
-\eta-\xi & -\beta-\eta-p \xi & -\beta-\eta-q \xi \\
0 & \beta-\varepsilon+p \xi-\xi & \beta+q \xi \\
0 & \varepsilon & -\gamma-\xi
\end{array}\right],
$$

Accordingly, the characteristic equation is easily obtained by the matrix (7) as

$$
\begin{aligned}
& (\lambda+\eta+\xi)\left[\lambda^{2}+\lambda(\gamma+\xi-\beta+\varepsilon+\xi-p \xi)+\left(\gamma \varepsilon+\varepsilon \xi+\gamma \xi+\xi^{2}-\gamma \beta-\xi \beta-\varepsilon \beta-\gamma p \xi-\varepsilon q \xi-p \xi^{2}\right)\right]=0, \\
& \lambda_{1}=-\eta-\xi \\
& \lambda_{2}=\frac{(\beta-\gamma-2 \xi-\varepsilon+p \xi)}{2}+\frac{\sqrt{(\gamma+2 \xi-\beta+\varepsilon-p \xi)^{2}}}{2}-\frac{\sqrt{4 *\left(\gamma \varepsilon+\varepsilon \xi+\gamma \xi+\xi^{2}-\gamma \beta-\xi \beta-\varepsilon \beta-\gamma p \xi-\varepsilon q \xi-p \xi^{2}\right)}}{2}, \\
& \lambda_{3}=\frac{(\beta-\gamma-2 \xi-\varepsilon+p \xi)}{2}-\frac{\sqrt{(\gamma+2 \xi-\beta+\varepsilon-p \xi)^{2}}}{2}-\frac{\sqrt{4 *\left(\gamma \varepsilon+\varepsilon \xi+\gamma \xi+\xi^{2}-\gamma \beta-\xi \beta-\varepsilon \beta-\gamma p \xi-\varepsilon q \xi-p \xi^{2}\right)}}{2} . \\
& \text { Clearly, } \lambda_{1}<0, \lambda_{3}<0, \text { and } \lambda_{2}<0 \text { if } R_{0} \leq 1, \text { which inti- } \\
& \text { mates } \beta(\xi+\varepsilon+\gamma)+p \xi(\gamma+\xi)+\varepsilon q \xi-(\gamma+\xi)(\varepsilon+\xi)<0 .
\end{aligned}
$$
The correctness of Theorem 1canbe proved according to stability theory in [36].

Theorem 2. If $R_{0} \leq 1, E_{0}$ is globally asymptotically stable relative to $\Psi$.

Proof. Give a Lyapunov function,
Obviously, the Lyapunov function is positive definite. Through direct computing, the differential equation of the function is obtained:

$$
\begin{aligned}
V \prime(t) & =\frac{d L}{d t}+\frac{\varepsilon-p \xi+q \xi+\xi}{(\xi+\gamma+\varepsilon)} \frac{d B}{d t}, \\
& =\left(\beta S-\frac{(\varepsilon+\xi)(\xi+\gamma)-p \xi(\xi+\gamma)-\varepsilon q \xi}{(\xi+\gamma+\varepsilon)}\right) L+\left(\beta S-\frac{(\varepsilon+\xi)(\xi+\gamma)-p \xi(\xi+\gamma)-\varepsilon q \xi}{(\xi+\gamma+\varepsilon)}\right) B .
\end{aligned}
$$


When $R_{0} \leq 1$, it is calculated that $\beta(\xi+\gamma+\varepsilon)+p \xi(\xi$ $+\gamma)+\varepsilon q \xi-(\xi+\gamma)(\varepsilon+\xi)<0$. It is observed that $V^{\prime}(t) \leq 0$. Furthermore, $V \prime(t)=0$ when and only when $L=0$ and $B=$ 0 are satisfied. In addition, when $L$ tends to be infinite or $B$ tends to infinity, one can get $V(t)$ to tend to be infinite. LaSalle's invariance principle [37] is an important basis for proving global stability. According to the theorem, we can see that when $R_{0} \leq 1$, the global stability of $E_{0}$ in $\Psi$ has been verified. Theorem 2 has been proved.

\section{Stability of the Virose Equilibrium}

Local stability and global stability analysis of endemic equilibrium is the important approach to analyze the propagation of the virus. At this stage, we put forward two theories to analyze the stability of $E^{*}$ and gave analysis and proof.

Theorem 3. In the case of $R_{0}>1$, the virose equilibrium is locally asymptotically stable.

Proof. We obtained the Jacobian matrix of system (3) at endemic equilibrium as follows:

$$
J_{E^{*}}=\left[\begin{array}{ccc}
-\beta\left(L^{*}+B^{*}\right)-\eta-\xi & -\beta S^{*}-\eta-p \xi & -\beta S^{*}-\eta-q \xi \\
\beta\left(L^{*}+B^{*}\right) & \beta S^{*}-\varepsilon+p \xi-\xi & \beta S^{*}+q \xi \\
0 & \varepsilon & -\gamma-\xi
\end{array}\right],
$$

The characteristic equation of $J_{E^{*}}$ is $\lambda^{3}+n_{1} \lambda^{2}+n_{2} \lambda+n_{3}=0$, where

$$
\begin{aligned}
n_{1} & =\eta+2 \xi+\gamma+H+A+B \\
n_{2} & =(\varepsilon+\eta+2 \xi+\gamma) H+\eta \gamma+\xi \gamma+\eta \xi+\xi^{2}+(\eta+\xi)(A+B), \\
n_{3} & =\left(\xi \gamma+\xi^{2}+\varepsilon \xi+\eta \gamma+\eta \xi+\varepsilon \eta+\gamma \varepsilon\right) H, \\
A & =q \xi \frac{\varepsilon}{\gamma+\xi} \\
B & =\beta S^{*} \frac{\varepsilon}{\gamma+\xi} \\
H & =\beta L^{*}+\beta B^{*} \\
\beta S^{*} & =\varepsilon+p \xi-A-B,
\end{aligned}
$$

we have

$$
\begin{aligned}
n_{1} n_{2}-n_{3}= & (\varepsilon+\gamma+\eta+2 \xi) H^{2}+(1+\varepsilon+\gamma+\eta+2 \xi)(A+B) H+H(\gamma+\xi)^{2}+H \varepsilon \xi+H(\xi+\eta)^{2}(\gamma+\xi) \\
& +(A+B)^{2}(\eta+\xi)+(A+B)(\xi+\eta)(\gamma+\xi)(1+\xi+\eta)+(\xi+\eta)(\gamma+\xi)(\gamma+\eta+2 \xi)
\end{aligned}
$$

By calculation, $n_{1}>0, n_{2}>0, n_{3}>0$, and $\left(n_{1} n_{2}-n_{3}\right)>0$ if $R_{0}>1$. On the basis of the Routh-Hurwitz criterion [38], Theorem 3 is proved.

Theorem 1 proves that the virus-free equilibrium is unstable under the condition of $R_{0}>1$. Besides that, as a result of $E_{0}$ belonging to the boundary of the feasible region
$\Psi$, state variables of the system are uniformly persistent $[39,40]$. Here, we advance the proposition as follows.

Proposition 1. System (3) is consistent and durable under the condition of $R_{0}>1$. In other words, there is a positive constant $\omega$ independent of the initial state of system (3), which satisfies

$$
\lim _{t \longrightarrow \infty} \text { in } f S(t)>\omega, \lim _{t \rightarrow \infty} \text { in } f L(t)>\omega, \lim _{t \longrightarrow \infty} \text { in } f B(t)>\omega, \lim _{t \longrightarrow \infty} \text { in } f(1-S(t)-L(t)-B(t))>\omega .
$$

Theorem 4. The virus equilibrium is globally asymptotically stable under the condition of $R_{0}>1$.

Proof. Now we will use a geometric method [41] to illustrate that $E^{*}$ is globally stable. From the above analysis, we know that system (3) has a compact attractive set $U \in \Psi^{*}$, and the only viral equilibrium exists in system (3).

Set $a=(S, L, B)$, and express the vector field of system (3) by $f(a)$. Find the Jacobian determinant, the Jacobian of the general solution $a(t)$ of system (3), that is, 
$J_{a(t)}=\left[\begin{array}{ccc}-\beta(L+B)-\eta-\xi & -\beta S-\eta-p \xi & -\beta S-\eta-q \xi \\ \beta(L+B) & \beta S-\varepsilon+p \xi-\xi & \beta S+q \xi \\ 0 & \varepsilon & -\gamma-\xi\end{array}\right]$.
By calculation, we obtain the second additive compound Jacobian matrix $[42,43]$ as

$$
J_{a(t)}^{[2]}=\left[\begin{array}{ccc}
-\beta(L+B-S)-(\eta+\xi+\varepsilon+p \xi) & \beta S+q^{\prime} \xi & \beta S+\eta-q \xi \\
\varepsilon & -\beta(L+B)-(\eta+2 \xi+\gamma) & -\beta S-\eta+p \xi \\
0 & \beta(L+B) & \beta S-(\gamma+\xi+\varepsilon+p \xi)
\end{array}\right] .
$$

Now, set the function $\Gamma(a)=\Gamma(S, L, B)$ as

$$
\Gamma(S, L, B)=\left[\begin{array}{ccc}
q_{1} & 0 & 0 \\
0 & \left(1-q_{2}\right) \frac{L}{B} & 0 \\
0 & q_{2} \frac{L}{B} & \frac{L}{B}
\end{array}\right],
$$

where

$$
\begin{gathered}
1<q_{1}<\frac{2 \beta \omega^{2}}{\beta+\xi+2 \eta}+1, \\
q_{2}= \begin{cases}0 & \varepsilon \geq \beta+\eta+p \xi, \\
1-\frac{\varepsilon}{\beta+\eta+p \xi} & \varepsilon<\beta+\eta+p \xi .\end{cases}
\end{gathered}
$$

Note that $\omega$ is the positive constant given in Proposition 1 . Then, there are

$$
\Gamma_{f} \Gamma^{-1}=\left[\begin{array}{ccc}
0 & 0 & 0 \\
0 & \frac{\dot{L}}{L}-\frac{\dot{B}}{B} & 0 \\
0 & 0 & \frac{\dot{L}}{L}-\frac{\dot{B}}{B}
\end{array}\right] .
$$

We rewrite the matrix $B=\Gamma_{f} \Gamma^{-} 1+\Gamma J_{a(t)}^{[2]} \Gamma^{-1}$ used in the geometric method of global stability [41]:

$$
B=\left[\begin{array}{ll}
B_{11} & B_{12} \\
B_{21} & B_{22}
\end{array}\right] \text {, }
$$

where

$$
\begin{aligned}
& B_{11}=[-\beta(L+B-S)-(\eta+2 \xi+\varepsilon-p \xi)], \\
& B_{12}=\left[\frac{q_{1} B(\beta S+q \xi)-q_{2} q_{1} B(\beta S+\eta+q \xi)}{\left(1-q_{2}\right) L} \frac{q_{1}(\beta S+\eta+q \xi) B}{L}\right], \\
& B_{21}=\left[\begin{array}{c}
\frac{\varepsilon\left(1-q_{2}\right) L}{q_{1} B} \\
\frac{\varepsilon q_{2} L}{q_{1} B}
\end{array}\right], \\
& B_{22}=\left[\begin{array}{cc}
\frac{\dot{L}}{L}-\frac{\dot{B}}{B}-\beta(L+B)-(\eta+2 \xi+\gamma)+q_{2}(\beta S+\eta+p \xi) & \left(q_{2}-1\right)(\beta S+\eta+p \xi) \\
\beta(L+B)+\frac{q_{2} \varepsilon}{1-q_{2}}-q_{2}(\beta S+\eta+p \xi) & \frac{\dot{L}}{L}-\frac{\dot{B}}{B}-(\gamma+2 \xi+\varepsilon+\eta)+\left(1-q_{2}\right)(\beta S+\eta+p \xi)
\end{array}\right] .
\end{aligned}
$$

The vectors in $R^{3}$ are denoted by $y=(\xi, \delta, \psi)$, where $|(\xi, \delta, \psi)|=\max |\xi|,|\delta|+|\psi|$ is selected as the norm of $R^{3}$.
Suppose that the Lozinskii measure about this norm is represented as $u$, we can obtain the following estimates [44]: 
$u(B) \leq \sup \left\{\kappa_{1}, \kappa_{2}\right\}=\sup \left\{u_{1}\left(B_{11}\right)+\left|B_{12}\right|, u_{1}\left(B_{22}\right)+\left|B_{21}\right|\right\}$,

where

$$
\begin{aligned}
& \kappa_{1}=u_{1}\left(B_{11}\right)+\left|B_{12}\right|, \\
& \kappa_{2}=u_{1}\left(B_{22}\right)+\left|B_{21}\right| .
\end{aligned}
$$

Accordingly, $u_{1}$ represents the Lozinskii measure of the norm of the $L_{1}$ vector, and $|B 21|,|B 12|$ are the matrix norms about $L_{1}$ vector norm.

Therefore, we obtain

$$
\begin{aligned}
& \kappa_{1}=-\beta(L+B-S)-(\eta+2 \xi+\varepsilon-p \xi)+\frac{q_{1}(\beta S+q \xi) B}{L}+\max \left\{\frac{-q_{1} q_{2} \eta B}{\left(1-q_{2}\right) L}, \frac{q_{1} \eta B}{L}\right\}, \\
& \kappa_{2}=\frac{\dot{L}}{L}-\frac{\dot{B}}{B}-(\eta+\gamma+2 \xi)+\frac{\varepsilon L}{q_{1} B}+\max \left\{q_{2}(\beta S+\eta+p \xi),-\varepsilon+2\left(1-q_{2}\right)(\beta S+\eta+p \xi)\right\},
\end{aligned}
$$

From (18) and (19), we can obtain

$$
\begin{aligned}
& \kappa_{1}=-\beta(L+B-S)-(\eta+2 \xi+\varepsilon-p \xi)+\frac{q_{1}(\beta S+q \xi) B}{L}+\frac{q_{1} \eta B}{L}, \\
& \kappa_{2} \leq \frac{\dot{L}}{L}-\frac{\dot{B}}{B}-(\eta+\gamma+2 \xi)+\eta+p \xi+\frac{\varepsilon L}{q_{1} B} .
\end{aligned}
$$

Now rewriting system (3), we have

$$
\begin{aligned}
& \frac{\dot{L}}{L}=\beta S+\beta S \frac{B}{L}-\varepsilon+p \xi-\xi+q \xi \frac{B}{L}, \\
& \frac{\dot{B}}{B}=\varepsilon \frac{L}{B}-\gamma-\xi .
\end{aligned}
$$

Adjust the consistent continuous constant $\omega$ in 4.1 so that there is a constant $T$ greater than zero in $K$ that is independent of the initial value $x(0)$ and satisfies

$$
S(t) \geq \omega, L(t) \geq \omega, B(t) \geq \omega \text { for } t>T .
$$

Substitute (28) into (26), and (29) into (27). From (18) and (19), using formula (27), we have, for $t>T$,

$$
\begin{aligned}
\kappa_{1} & =\frac{\dot{L}}{L}+\frac{\left(q_{1}-1\right)(\beta S+q \xi) B}{L}-\beta(L+B)-\xi-\eta+\frac{q_{1} \eta B}{L} \\
& \leq \frac{\dot{L}}{L}+\frac{\left(q_{1}-1\right)(\beta S+q \xi)}{\omega}-2 \beta \omega-\xi-\eta+\frac{q_{1} \eta}{\omega} \\
& \leq \frac{\dot{L}}{L}-\xi-\eta \\
& \leq \frac{\dot{L}}{L}-\xi \\
\kappa_{2} & \leq \frac{\dot{L}}{L}-\frac{\varepsilon L}{B}+\frac{\varepsilon L}{q_{1} B} \\
& \leq \frac{\dot{L}}{L}-\frac{\left(q_{1}-1\right) \varepsilon L}{q_{1} B} .
\end{aligned}
$$

Consequently, $u(B) \leq \dot{L} / L-b$ for $t>T$ by (23), and (31) and (32), where $b=\min \left\{\xi,\left(q_{1}-1\right) \varepsilon L / q_{1} B\right\}>0$. For each answer $x\left(t, x_{0}\right)$ of system (3) satisfying $x_{0} \in K$ and $t>T$, there is

$$
\frac{1}{t} \int_{0}^{T} u(B) \mathrm{d} s \leq \frac{1}{t} \int_{0}^{T} u(B) \mathrm{d} s+\frac{1}{t} \log \frac{L(t)}{L(T)}-b \frac{t-T}{t},
$$

meaning that $\bar{q} \leq-b / 2<0$ from (23), proving Theorem 4 .

\section{Further Discussion}

Combined with the analysis in the previous sections, we first analyze the sensitivity of $R_{0}$ to system parameters and give a visualization diagram. Then, we studied the influence of relevant parameters on $R_{0}$ and drew a series of visualization graphs.

As described earlier, $\beta$ denotes the infection rate of uninfected computers, and $p$ and $q$ are part of infected newborns in the latent internal computers and breaking-out internal computers classes, respectively. In addition, $\gamma$ represents the healing rate of disconnected nodes. To better analyze the spread of computer viruses, it is necessary to carry out sensitivity analysis of these system parameters for $R_{0}$. Next, calculate the normalized forward sensitivity index of $\beta, p, q$, and $\gamma$ [45] as follows, respectively:

$\frac{\partial R_{0} / R_{0}}{\partial \beta / \beta}=\frac{\beta}{R_{0}} \frac{\partial R_{0}}{\partial \beta}=\frac{\beta(\xi+\gamma+\varepsilon)}{\beta(\xi+\gamma+\varepsilon)+(\varepsilon+\gamma) p \xi+\varepsilon q \xi}>0$,

$\frac{\partial R_{0} / R_{0}}{\partial p / p}=\frac{p(\xi+\gamma)}{\beta(\xi+\gamma+\varepsilon)+(\varepsilon+\gamma) p \xi+\varepsilon q \xi}>0$,

$\frac{\partial R_{0} / R_{0}}{\partial q / q}=\frac{q \xi \varepsilon}{\beta(\xi+\gamma+\varepsilon)+(\varepsilon+\gamma) p \xi+\varepsilon q \xi}>0$,

$\frac{\partial R_{0} / R_{0}}{\partial \gamma / \gamma}=\frac{-\gamma(\beta \varepsilon+\varepsilon q \xi)}{(\xi+\gamma)[\beta(\xi+\gamma+\varepsilon)+(\varepsilon+\gamma) p \xi+\varepsilon q \xi]}<0$.

From these numerical results, we can draw the following conclusions: 

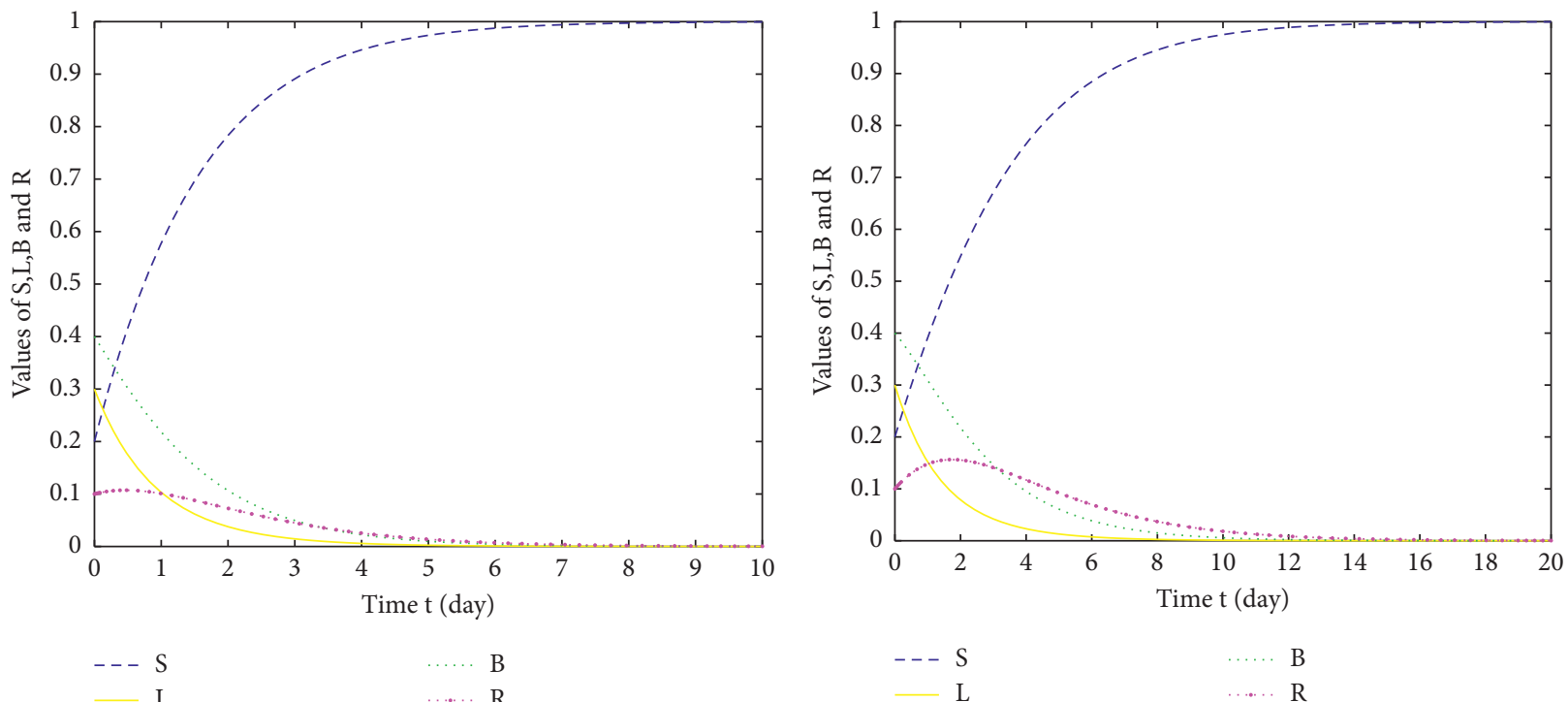

(a)

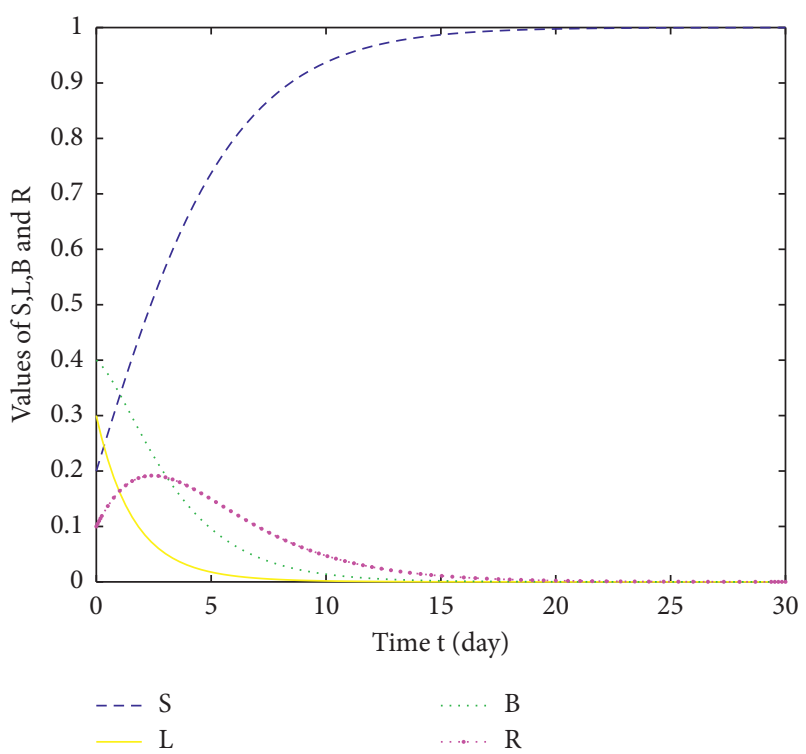

(c)

(b)

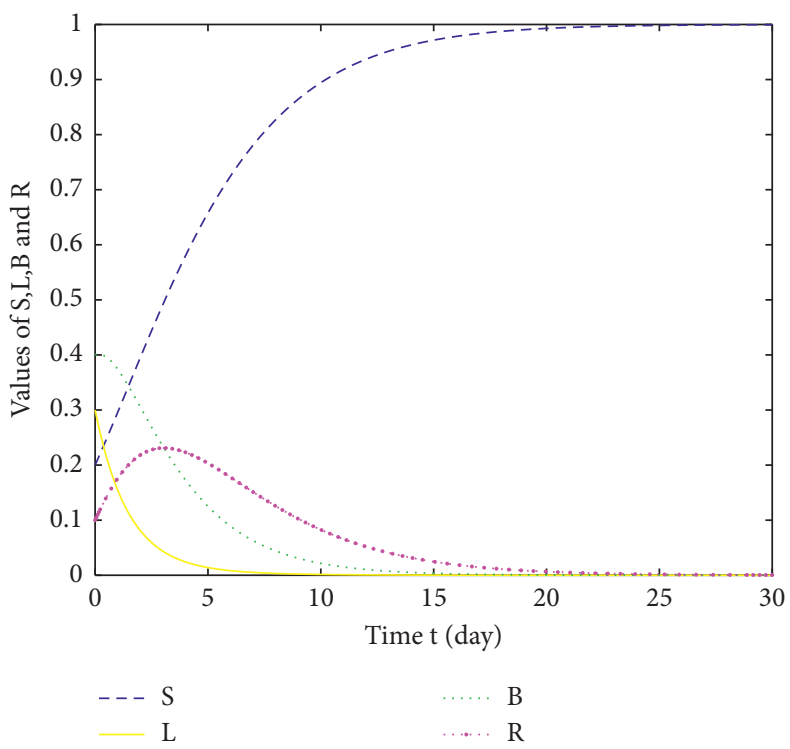

(d)

Figure 2: Evolution trend chart of system (3) at (a) $R_{0}=0.3119$, (b) $R_{0}=0.5242$, (c) $R_{0}=0.6783$, and (d) at $R_{0}=0.8333$.

TABle 3: The typical $R_{0}$ and associated parameter values of system (3).

\begin{tabular}{lcccccccccc}
\hline$R_{0}$ & $\xi$ & $\beta$ & $\eta$ & $p$ & $q$ & $\gamma$ & $\epsilon$ & $S(0)$ & $L(0)$ & $B(0)$ \\
\hline 0.3119 & 0.66 & 0.01 & 0.2 & 0.1 & 0.1 & 0.3 & 0.6 & 0.2 & 0.3 & 0.4 \\
0.5242 & 0.26 & 0.01 & 0.2 & 0.1 & 0.1 & 0.3 & 0.5 & 0.2 & 0.3 & 0.4 \\
0.6783 & 0.16 & 0.01 & 0.2 & 0.1 & 0.1 & 0.3 & 0.5 & 0.2 & 0.3 & 0.4 \\
0.8333 & 0.1 & 0.01 & 0.2 & 0.1 & 0.1 & 0.3 & 0.6 & 0.2 & 0.3 & 0.4 \\
\hline
\end{tabular}

(1) If the infection rate $\beta$ is reduced, the spread of the virus can be controlled.
(2) If the birth rate of nodes in the vertical direction, $p, q$, is reduced, it will help control the spread of the virus. 


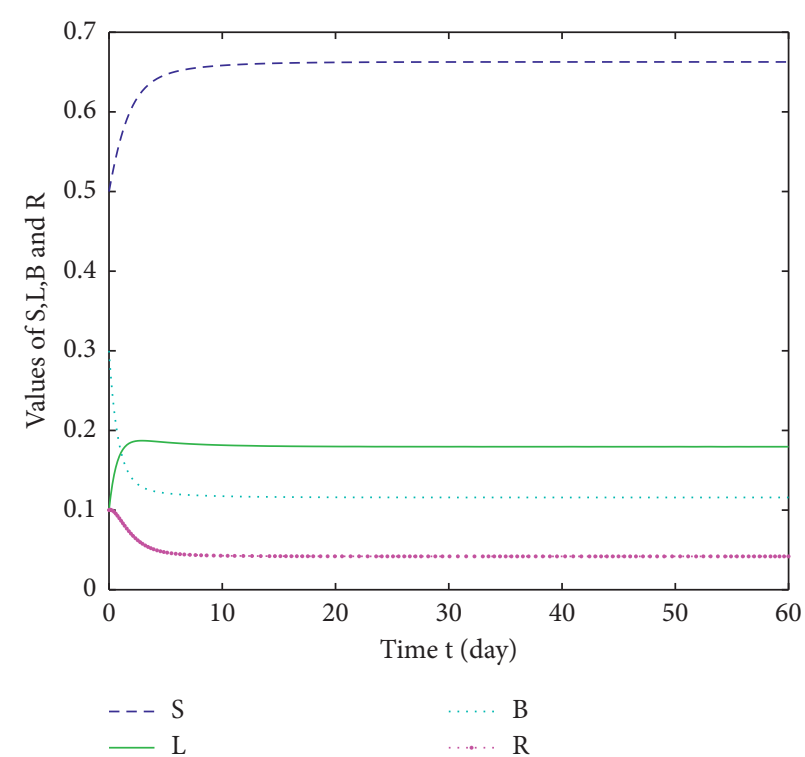

(a)

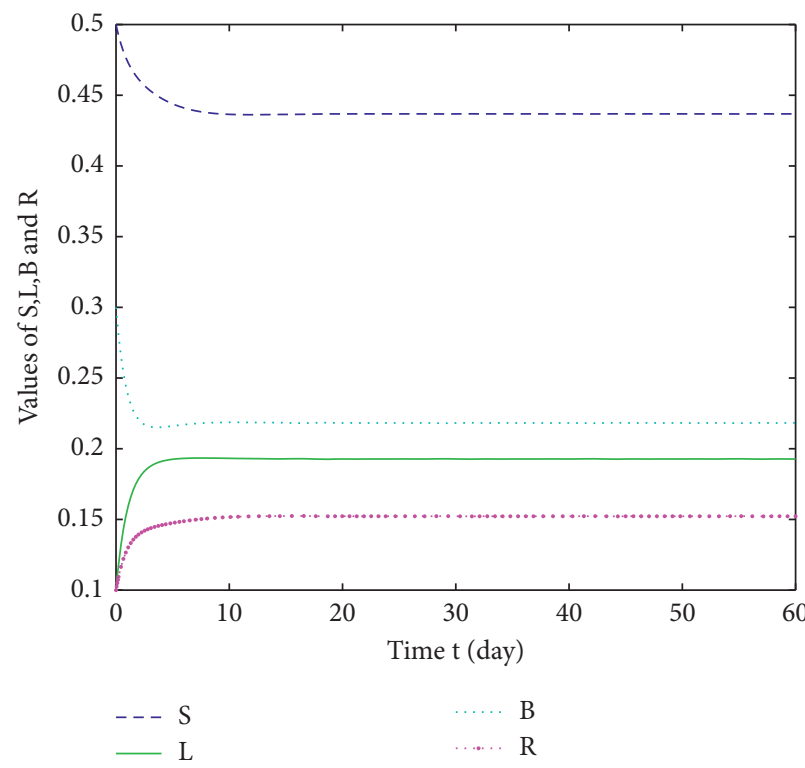

(c)

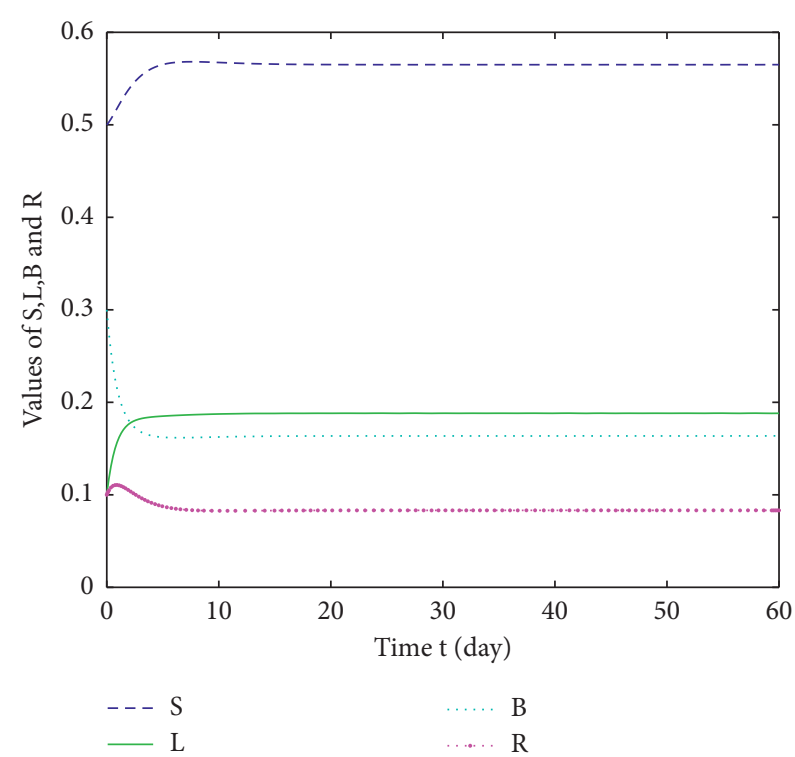

(b)

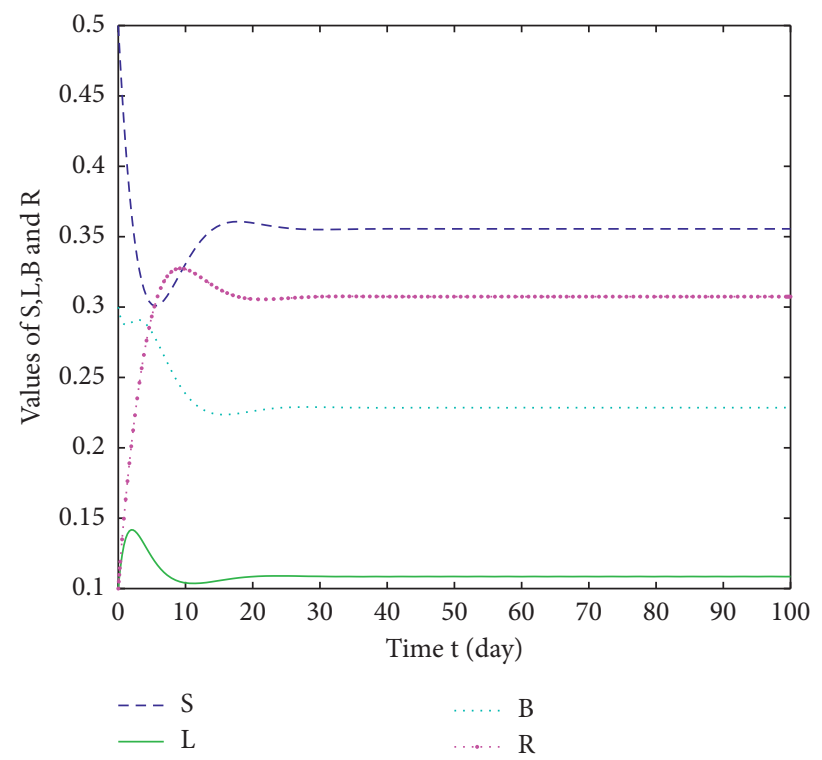

(d)

Figure 3: Evolution trend chart of system (3) at (a) $R_{0}=3.9880$, (b) $R_{0}=6.1771$, (c) $R_{0}=11.2353$, and (d) $R_{0}=121.7206$.

(3) If the cure rate $\gamma$ is increased, it will help suppress the virus.

\section{Numerical Experiments}

Numerical simulations are an important tool for the quantitative analysis of models. Next, a series of numerical examples were made to visualize all the above theories.

6.1. Stability Analysis. For $R_{0} \leq 1, E_{0}$ is globally asymptotically stable according to Theorem 2 . Figure 2 shows how the various types of states in the network evolve in time for $R_{0}=0.3119,0.5242,0.6783$ and 0.8333 where the corresponding parameter values are shown in Table 3 . From the graph, we can see that if $R_{0} \leq 1$, the virus in the network eventually tends to die out.

For $R_{0}>1$, after the previous analysis and elaboration, Theorem 4 has been confirmed. Figure 3 shows how the various types of states in the network evolve in time for $R_{0}=3.9880,6.1771,11.2353$, and 121.7206 , where the corresponding parameter values are shown in Table 4. From the graph, we can see that if $R_{0}>1$, the virus in the network will always be there and tend to stabilizes. 
TABLE 4: The typical $R_{0}$ and associated parameter values of system (3).

\begin{tabular}{lcccccccccc}
\hline $11 \mathrm{~F} 0 \mathrm{E} 0 R_{0}$ & $\xi$ & $\beta$ & $\eta$ & $p$ & $\epsilon$ & $\gamma$ & $q$ & $S(0)$ & $L(0)$ & $B(0)$ \\
\hline 3.9880 & 0.63 & 0.5 & 0.2 & 0.7 & 0.6 & 0.3 & 0.6 & 0.5 & 0.1 & 0.3 \\
6.1771 & 0.39 & 0.56 & 0.2 & 0.5 & 0.6 & 0.3 & 0.6 & 0.5 & 0.1 & 0.3 \\
11.2353 & 0.23 & 0.6 & 0.2 & 0.5 & 0.6 & 0.3 & 0.6 & 0.5 & 0.1 \\
121.7206 & 0.023 & 0.6 & 0.2 & 0.5 & 0.6 & 0.3 & 0.68 & 0.5 & 0.1 & 0.3 \\
\hline
\end{tabular}

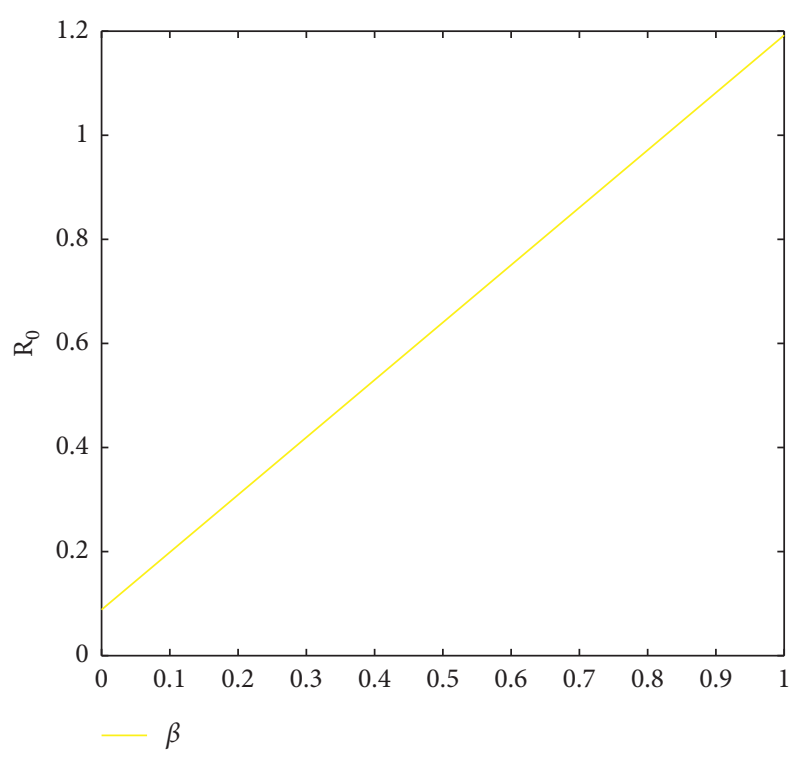

FIgURE 4: Influence of parameter $\beta$ on $R_{0}$.

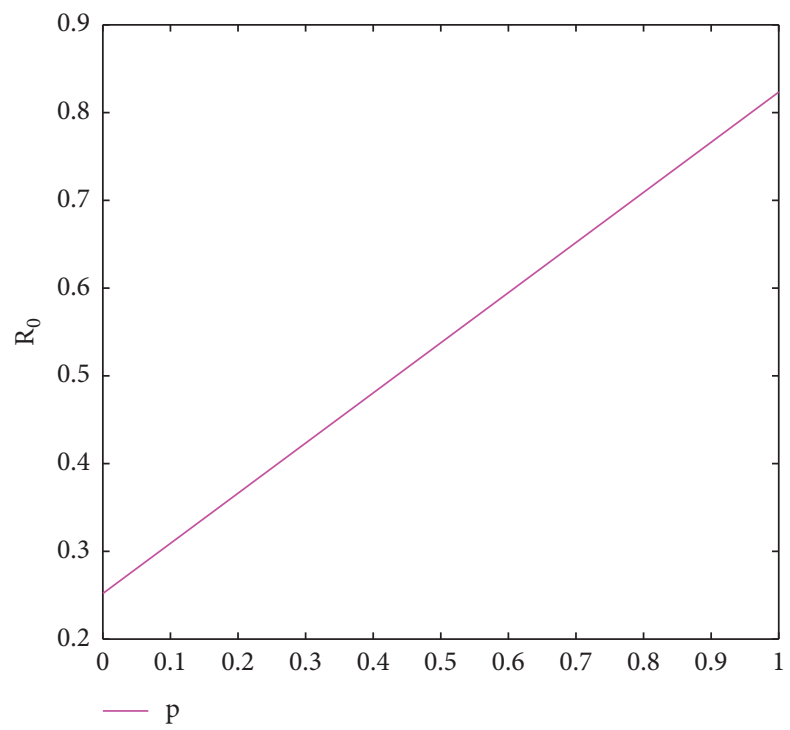

FIgURE 5: Influence of parameter $p$ on $R_{0}$.

6.2. Influence of Parameters on the Value of $R_{0}$. From the previous subsection, we can see that the basic regeneration number determines whether the virus tends to die out in the network or not. So analyzing the effect of parameters in the system on the basic regeneration number can help to contain the spread of the virus. It can 


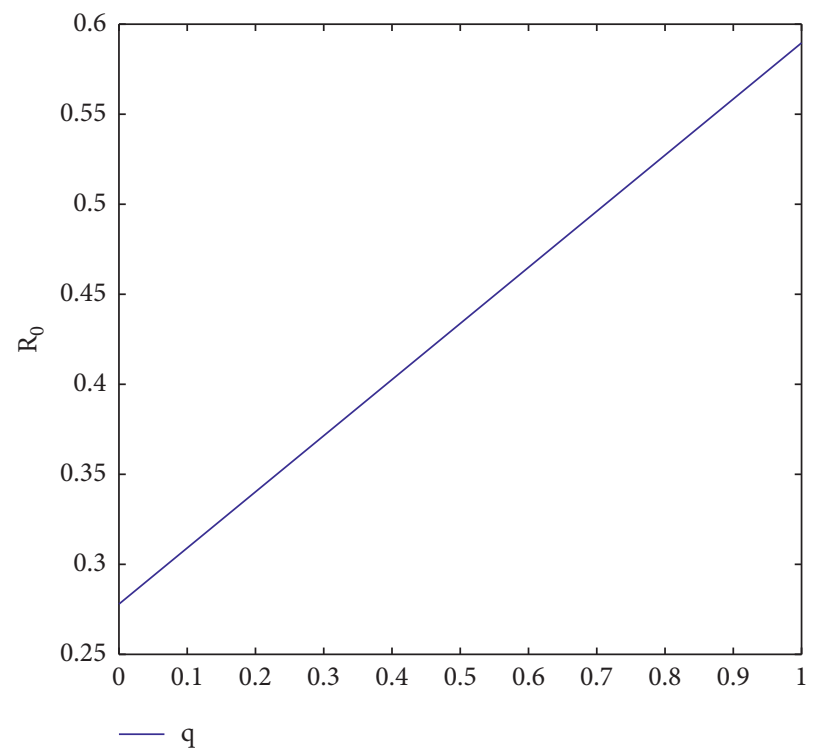

FIgURE 6: Influence of parameter $q$ on $R_{0}$.

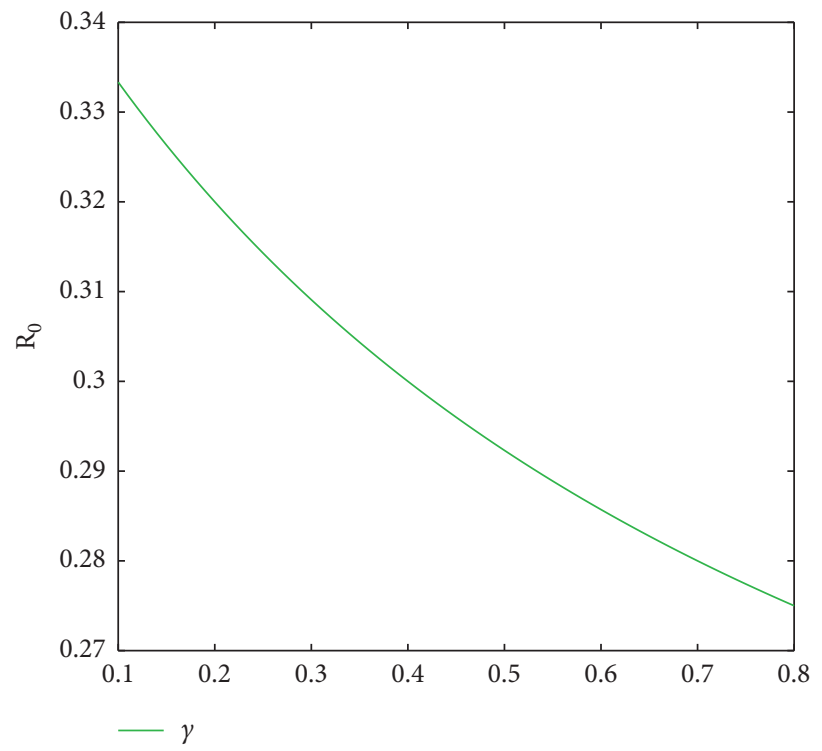

Figure 7: Influence of parameter $\gamma$ on $R_{0}$.

TABLE 5: Simulation parameters.

\begin{tabular}{lccccccc}
\hline Figure & $\xi$ & $\beta$ & $\eta$ & $p$ & $q$ & $\gamma$ \\
\hline Figure 4 & 0.8 & $*$ & 0.2 & 0.1 & 0.1 & 0.3 \\
Figure 5 & 0.8 & 0.2 & 0.2 & $*$ & 0.1 & 0.3 \\
Figure 6 & 0.8 & 0.2 & 0.2 & 0.1 & $*$ & 0.3 \\
Figure 7 & 0.8 & 0.2 & 0.1 & 0.1 & 0.3 & 0.6 \\
\hline
\end{tabular}

be seen that Figures $4-6$ show that increases in $\beta$, $p$, and $q$ produce an equal proportional increase in $R_{0}$, respectively. Figure 7 shows a decline in the size of $R_{0}$ as the parameter $\gamma$ increases. The parameters are shown in Table 5. These numerical simulations validate the analysis in Section 5. 


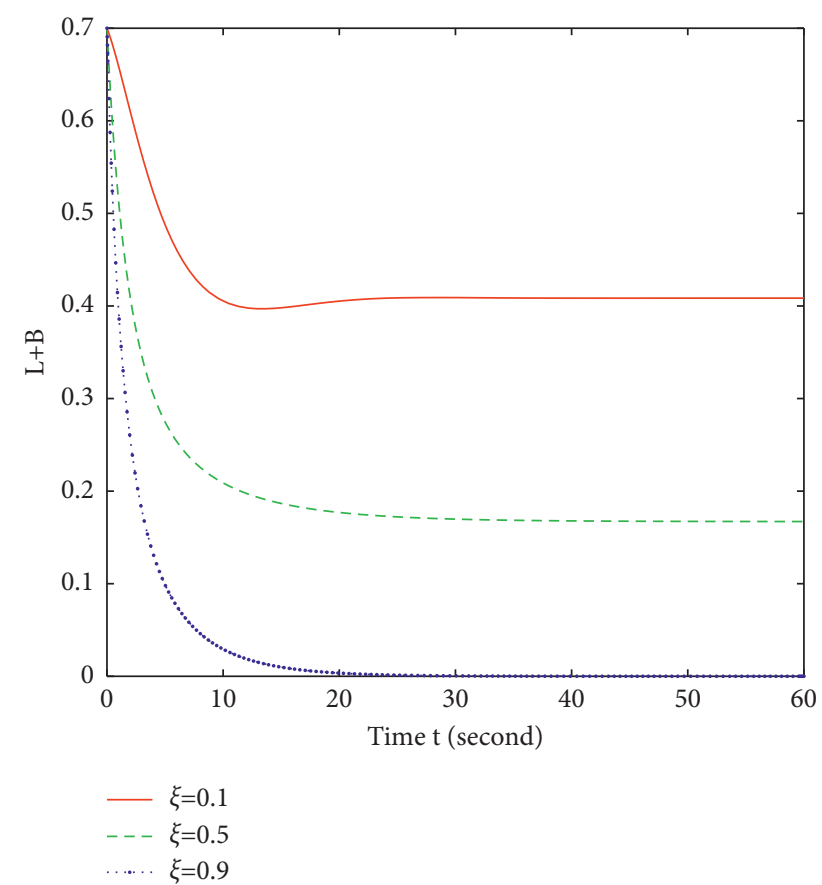

Figure 8: Influence of parameter $\xi$ on $L+B$.

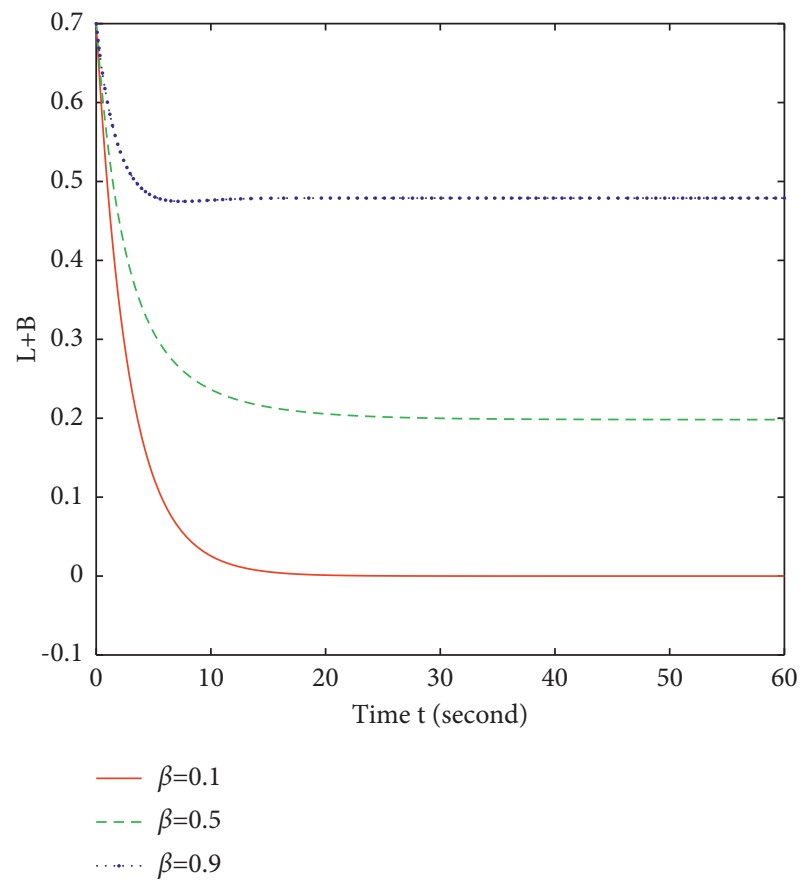

FIGURE 9: Influence of parameter $\beta$ on $L+B$.

6.3. Influence of Parameters on the Number of Infected Nodes. When the basic regeneration number is inevitably greater than one, we take steps to reduce the number of infected

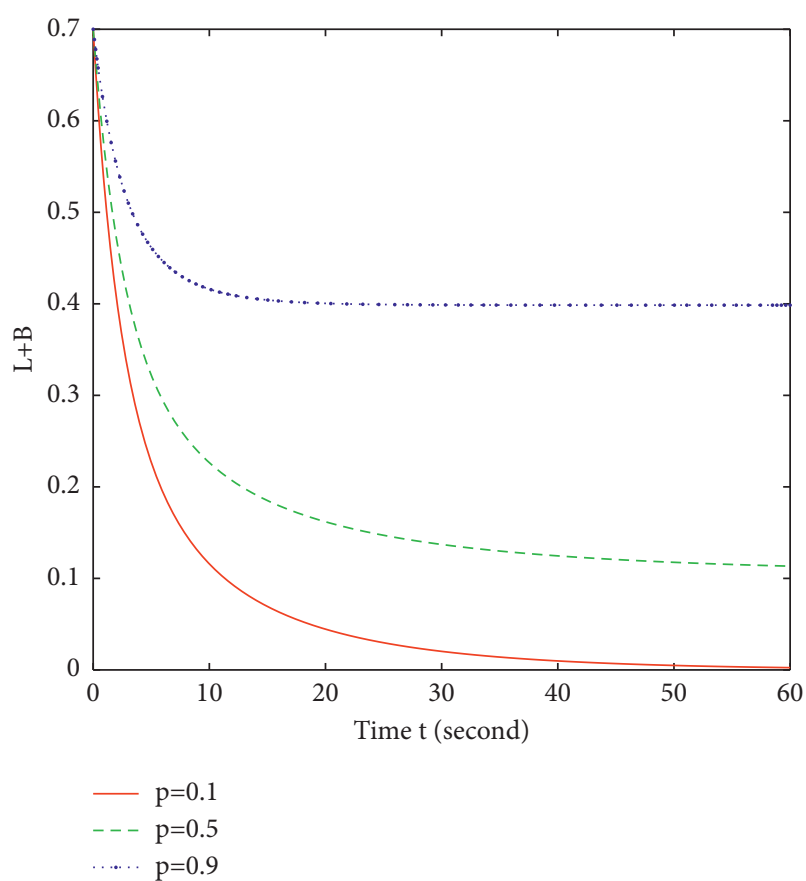

Figure 10: Influence of parameter $p$ on $L+B$.

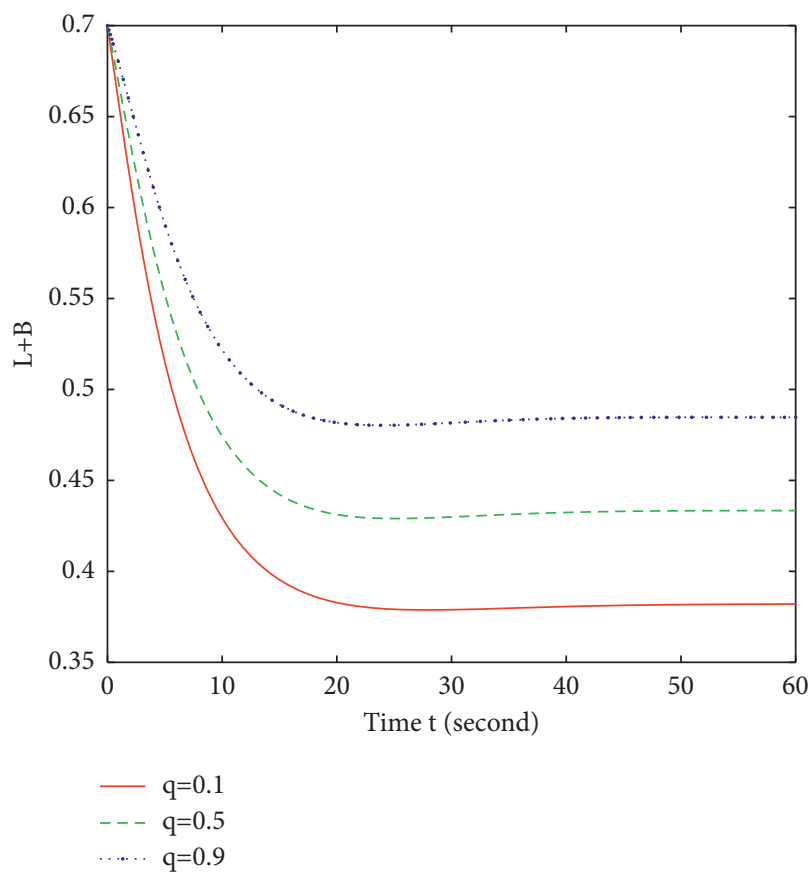

FIGURE 11: Influence of parameter $q$ on $L+B$.

nodes. Analyzing the impact of parameters on infected nodes helps us to select measures to contain the virus. In this subsection, we analyze the parameters that affect the level of 


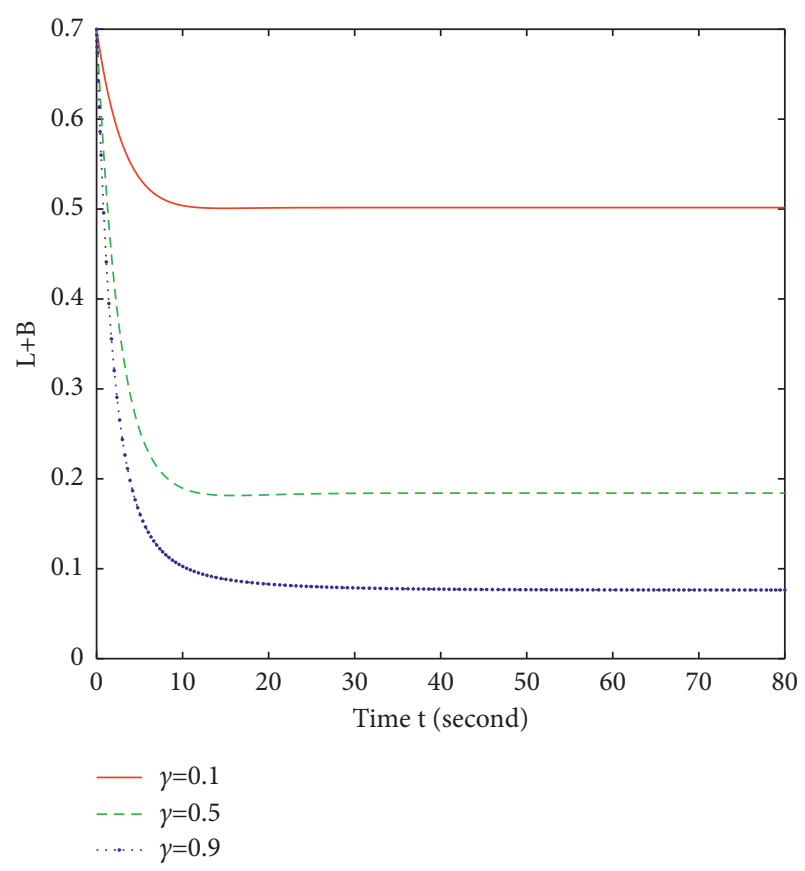

Figure 12: Influence of parameter $\gamma$ on $L+B$.

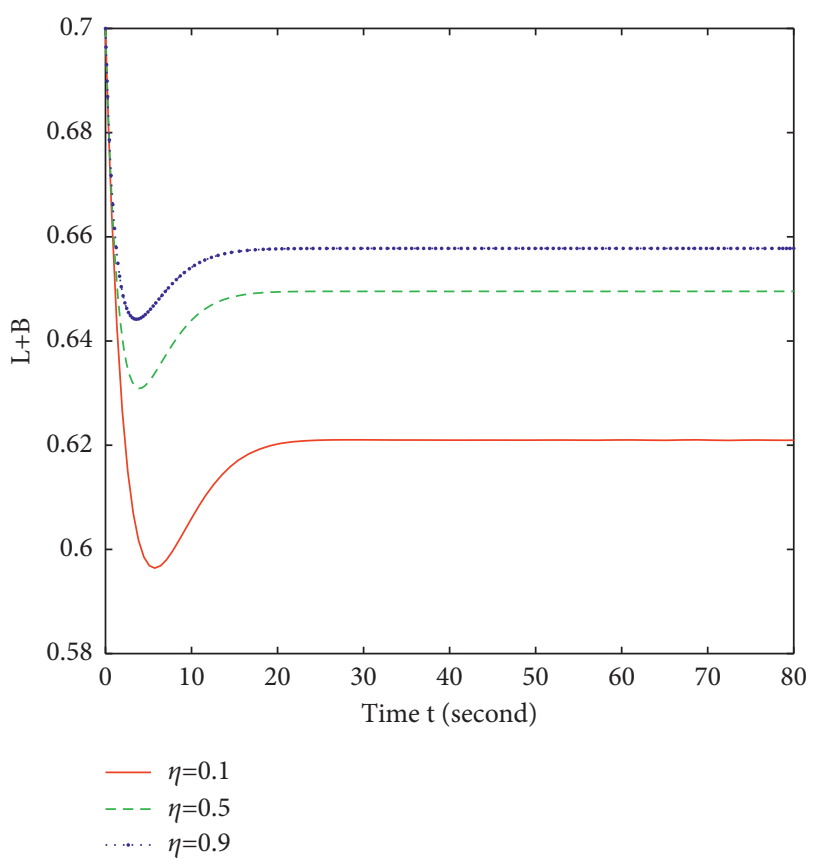

FIGURE 13: Influence of parameter $\eta$ on $L+B$.

stability of the infected computer, and Figures 8-13 show the results of the analysis.

\section{Conclusion}

Considering the impact of the vertical transmission of computer viruses on new uninfected nodes, a novel dynamic model of network virus transmission is put forward, and this model is discussed and analyzed. The conclusions drawn from the analysis of the model are as follows:
(1) This article studies an SLBRS model in which the virus infection can spread vertically and horizontally

(2) It is confirmed that this model is asymptotically stable globally

(3) Numerical simulation verifies the theoretical analysis of model balance

(4) Perform system parameter sensitivity analysis on $R_{0}$

(5) The parameters affecting the stability level of the infected computer are analyzed

To our minds, the model proposed in this article more truly reflects some of the characteristics of network viruses than previous models. We believe that in the later work, the model can be extended on the complex network, and consider some of the most optimal control strategies.

\section{Abbreviations}

SEI: $\quad$ Susceptible-exposed-infected

SEIQRS: Susceptible-exposed-infected-quarantinedrecovered-susceptible

SEIQV: Susceptible-exposed-infected-quarantinedvaccinated

SEIR: Susceptible-exposed-infected-recovered

SEIRS: Susceptible-exposed-infected-recoveredsusceptible

SIR: $\quad$ Susceptible-infected-recovered

SIRS: $\quad$ Susceptible-infected-recovered-susceptible

SIS: $\quad$ Susceptibility-infection-susceptibility

SLBRS: Susceptible-latent-breaking-recoveredsusceptible

SLBS: Susceptible-latent-breaking-susceptible.

\section{Data Availability}

Data sharing is not applicable to this article as no datasets were generated.

\section{Conflicts of Interest}

All authors declare that there are no conflicts of interest.

\section{Authors' Contributions}

The authors claim that the research was realized in collaboration with the same responsibility. All authors read and approved the last version of the manuscript.

\section{Acknowledgments}

This work was supported by the National Natural Science Foundation of China (Grant no. 61903056), Major Project of Science and Technology Research Program of Chongqing Education Commission of China (Grant no. KJZDM201900601), Chongqing Research Program of Basic Research and Frontier Technology (Grant nos. cstc2019jcyj-msxmX0681, cstc2021jcyjmsxmX0530, and cstc2021jcyj-msxmX0761), Chongqing Municipal Key Laboratory of Institutions of Higher Education (Grant no. cqupt-mct-201901), Chongqing Key Laboratory of 
Mobile Communications Technology (Grant no. cqupt-mct202002), and Engineering Research Center of Mobile Communications, Ministry of Education (Grant no. cquptmct202006).

\section{References}

[1] M. Shoaib Arif, A. Raza, M. Rafiq et al., "Numerical simulations for stochastic computer virus propagation model," Computers, Materials \& Continua, vol. 62, no. 1, pp. 61-77, 2020.

[2] X. Han and Q. Tan, "Dynamical behavior of computer virus on internet," Applied Mathematics and Computation, vol. 217, no. 6, pp. 2520-2526, 2010.

[3] N. Scaife, H. Carter, P. Traynor, and K. R. Butler, "Cryptolock (and drop it): stopping ransomware attacks on user data," in Proceedings of the 2016 IEEE 36th International Conference on Distributed Computing Systems (ICDCS), pp. 303-312, IEEE, Nara, Japan, June 2016.

[4] krebsonsecurity, Parkmobile Breach Exposes License Plate Data, mobile Numbers of $21 \mathrm{~m}$ Users, https://krebsonsecurity. com/2021/04/parkmobile-breach-exposes-license-plate-datamobile-numbers-of-21m-users/, accessed April 12, 2021, 2021.

[5] J. O. Kephart and S. R. White, "Directed-graph epidemiological models of computer viruses," in Proceedings of the 1991 IEEE Computer Society Symposium on Research in Security and Privacy, pp. 71-102, IEEE, Oakland, CA, USA, May 1992.

[6] B. Xie and M. Liu, "Dynamics stability and optimal control of virus propagation based on the e-mail network," IEEE Access, vol. 9, pp. 32449-32456.

[7] V. P. Dubey, R. Kumar, and D. Kumar, "A hybrid analytical scheme for the numerical computation of time fractional computer virus propagation model and its stability analysis," Chaos, Solitons \& Fractals, vol. 133, Article ID 109626, 2020.

[8] M. Zhang, K. Liu, K. Liu, L. Chen, and Z. Li, "State feedback impulsive control of computer worm and virus with saturated incidence," Mathematical Biosciences and Engineering, vol. 15, no. 6, pp. 1465-1478, 2018.

[9] D. Prodanov, "Comments on some analytical and numerical aspects of the sir model," Applied Mathematical Modelling, vol. 95, pp. 236-243, 2021.

[10] H. El-Saka, A. Arafa, and M. Gouda, "Dynamical analysis of a fractional sirs model on homogenous networks," Advances in Difference Equations, vol. 2019, no. 1, pp. 1-15, 2019.

[11] B. Li and Q. Bie, "Long-time dynamics of an sirs reactiondiffusion epidemic model," Journal of Mathematical Analysis and Applications, vol. 475, no. 2, pp. 1910-1926, 2019.

[12] S. P. Rajasekar and M. Pitchaimani, "Ergodic stationary distribution and extinction of a stochastic sirs epidemic model with logistic growth and nonlinear incidence," Applied Mathematics and Computation, vol. 377, Article ID 125143, 2020.

[13] Q. Liu, D. Jiang, and D. Jiang, "Dynamics of a multigroup sirs epidemic model with random perturbations and varying total population size," Communications on Pure and Applied Analysis, vol. 19, no. 2, pp. 1089-1110, 2020.

[14] S. Kim, J. H. Byun, and I. H. Jung, "Global stability of an seir epidemic model where empirical distribution of incubation period is approximated by coxian distribution," Advances in Difference Equations, vol. 2019, no. 1, pp. 1-15, 2019.

[15] S. Han and C. Lei, "Global stability of equilibria of a diffusive seir epidemic model with nonlinear incidence," Applied Mathematics Letters, vol. 98, pp. 114-120, 2019.
[16] Y. Yang and L. Xu, "Stability of a fractional order seir model with general incidence," Applied Mathematics Letters, vol. 105, Article ID 106303, 2020.

[17] H. Kang, M. Sun, Y. Yu, X. Fu, and B. Bao, "Spreading dynamics of an seir model with delay on scale-free networks," IEEE Transactions on Network Science and Engineering, vol. 7, no. 1, pp. 489-496, 2018.

[18] Z. Yin, Y. Yu, and Z. Lu, "Stability analysis of an agestructured seirs model with time delay," Mathematics, vol. 8, no. 3, p. 455, 2020.

[19] L. Zhang, X. Fan, and Z. Teng, "Global dynamics of a nonautonomous seirs epidemic model with vaccination and nonlinear incidence," Mathematical Methods in the Applied Sciences, vol. 44, no. 11, pp. 9315-9333, 2021.

[20] P. Song, Y. Lou, and Y. Xiao, "A spatial seirs reaction-diffusion model in heterogeneous environment," Journal of Differential Equations, vol. 267, no. 9, pp. 5084-5114, 2019.

[21] W. Yang, "Dynamical behaviors and optimal control problem of an seirs epidemic model with interventions," Bulletin of the Malaysian Mathematical Sciences Society, vol. 44, no. 2, pp. 1-16, 2021.

[22] M. A. Khan, Y. Khan, T. W. Khan, and S. Islam, "Dynamical system of a seiqv epidemic model with nonlinear generalized incidence rate arising in biology," International Journal of Biomathematics, vol. 10, no. 7, Article ID 1750096, 2017.

[23] N. Ahmed, M. Fatima, D. Baleanu et al., "Numerical analysis of the susceptible exposed infected quarantined and vaccinated (seiqv) reaction-diffusion epidemic model," Frontiers in Physics, vol. 7, p. 220, 2020.

[24] Z. Zhang and L. Song, "Dynamics of a computer virus propagation model with delays and graded infection rate," Advances in Mathematical Physics, vol. 2017, no. 1, 13 pages, Article ID 4514935, 2017.

[25] Z. Zhang, S. Kundu, and R. Wei, "A delayed epidemic model for propagation of malicious codes in wireless sensor network," Mathematics, vol. 7, no. 5, p. 396, 2019.

[26] B. K. Mishra and N. Jha, "Seiqrs model for the transmission of malicious objects in computer network," Applied Mathematical Modelling, vol. 34, no. 3, pp. 710-715, 2010.

[27] M. Essouifi and A. Achahbar, "A mixed sir-sis model to contain a virus spreading through networks with two degrees," International Journal of Modern Physics C, vol. 28, no. 9, Article ID 1750114, 2017.

[28] R. K. Upadhyay, S. Kumari, and A. K. Misra, "Modeling the virus dynamics in computer network with sveir model and nonlinear incident rate," Journal of Applied Mathematics and Computing, vol. 54, no. 1, pp. 485-509, 2016.

[29] F. Yang and Z. Zhang, "Hopf bifurcation analysis of seir-ks computer virus spreading model with two-delay," Results in Physics, vol. 24, no. 1, Article ID 104090, 2021.

[30] A. Coronel, F. Huancas, I. Hess, E. Lozada, and F. NovoaMuoz, "Analysis of a Seir-Ks mathematical model for computer virus propagation in a periodic environment," Mathematics, vol. 8, no. 5, p. 20, 2020.

[31] N. Zdemir, S. Uar, and B. B. S. Erolu, "Dynamical analysis of fractional order model for computer virus propagation with kill signals," International Journal of Nonlinear Sciences and Numerical Stimulation, vol. 21, no. 3-4, pp. 239-247, 2020.

[32] W. Tang, Y.-J. Liu, Y.-L. Chen, Y.-X. Yang, and X.-X. Niu, "Slbrs: network virus propagation model based on safety entropy," Applied Soft Computing, vol. 97, Article ID 106784, 2020. 
[33] X. Yang and L.-X. Yang, "Towards the epidemiological modeling of computer viruses," Discrete Dynamics in Nature and Society, vol. 2012, Article ID 259671, 11 pages, 2012.

[34] M. Yang, Z. Zhang, Q. Li, and G. Zhang, "An slbrs model with vertical transmission of computer virus over the internet," Discrete Dynamics in Nature and Society, vol. 2012, Article ID 925648, 17 pages, 2012.

[35] B. K. Mishra and S. K. Pandey, "Dynamic model of worms with vertical transmission in computer network," Applied Mathematics and Computation, vol. 217, no. 21, pp. 8438-8446, 2011.

[36] J. K. Hale, Theory of Functional Differential Equation, Springer, New York, NY, USA, 1977.

[37] J. P. La Salle, The Stability of Dynamical Systems, SIAM, Thailand, 1976.

[38] R. C. Robinson, An Introduction to Dynamical Systems, American Mathematical Society, RI, USA, 2005.

[39] H. I. Freedman, S. Ruan, and M. Tang, "Uniform persistence and flows near a closed positively invariant set," Journal of Dynamics and Differential Equations, vol. 6, no. 4, pp. 583-600, 1994.

[40] S. Sun, "Global dynamics of a seir model with a varying total population size and vaccination," International Journal of Mathematics and Analysis, vol. 6, no. 40, pp. 1985-1995, 2012.

[41] M. Y. Li and J. S. Muldowney, "A geometric approach to global-stability problems," SIAM Journal on Mathematical Analysis, vol. 27, no. 4, pp. 1070-1083, 1996.

[42] M. Fiedler, "Additive compound matrices and an inequality for eigenvalues of symmetric stochastic matrices," Czechoslovak Mathematical Journal, vol. 24, no. 3, pp. 392-402, 1974.

[43] J. S. Muldowney, "Compound matrices and ordinary differential equations," Rocky Mountain Journal of Mathematics, vol. 20, no. 4, pp. 857-872, 1990.

[44] R. H. Martin Jr., "Logarithmic norms and projections applied to linear differential systems," Journal of Mathematical Analysis and Applications, vol. 45, no. 2, pp. 432-454, 1974.

[45] L. Arriola and J. Hyman, "Forward and adjoint sensitivity analysis with applications in dynamical systems," Lecture Notes in Linear Algebra and Optimization, Mathematical and Theoretical Biology Institute, Columbus, OH, USA, 2005. 